\title{
Génomique et bioterrorisme
}

Maurice Boissinot, Michel G. Bergeron

$>$ Les attaques terroristes récentes utilisant des spores de Bacillus anthracis ont révélé les lacunes des organismes de santé publique. Pourtant, l'utilisation des bactéries comme arme n'est pas nouvelle et il faudra développer notre capacité à y faire face. II est important de mettre au point de nouvelles méthodes de diagnostic moléculaire afin d'identifier les bactéries en moins d'une heure. Notre laboratoire utilise la génomique pour créer des outils qui promettent de révolutionner la médecine. <

\section{Les armes biologiques dans l'histoire}

Les lettres contaminées au bacille du charbon à l'automne 2001 ne sont pas les premières attaques réalisées avec des armes biologiques. En fait, dès que l'être humain a été en mesure d'observer le caractère transmissible de certaines maladies, il a utilisé son savoir à des fins belliqueuses. Dans l'Antiquité, il s'agissait de stratégies simples, comme contaminer les puits des ennemis avec des cadavres d'animaux. Les connaissances sur les infections se sont par la suite approfondies ; par exemple, dans le troisième chant de ses Géorgiques, Virgile présente une très vivante et poétique description de la maladie du charbon chez les animaux et de sa transmission à l'homme par le port de lainages et de peaux contaminés. Plus tard, Koch a formulé ses célèbres postulats à la suite de ses observations sur la transmission de cette infection. Au XIV siècle, les Tatars ont tenté de répandre la peste dans la ville de Kaffa assiégée en catapultant des cadavres de pestiférés par dessus les remparts. Au cours de la révolte menée par le chef amérindien Pontiac (1763), le commandant anglais Sir Jeffrey Amherst avait fait distribuer des couvertures de varioleux aux populations amérindiennes. Pendant la Première Guerre mondiale, l'Allemagne a réalisé de nombreuses attaques biologiques sur les

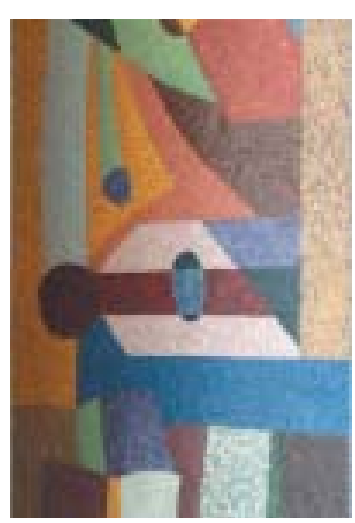

Alliés. Un espion allemand a même tenté de contaminer des troupeaux de rennes qui servaient de bêtes de somme pour le transport d'armes entre la Norvège et la Russie. Les spores de Bacillus anthracis saisies sur lui ont pu être réanimées après plus de quatre-vingts ans d'entreposage dans les locaux du musée de la police de Trondheim [1]. Au cours de la Seconde Guerre mondiale, les Alliés ont mis au point plusieurs armes bactériologiques dont le développement s'est accéléré pendant la guerre froide, surtout aux États-Unis et en Union soviétique [2].

\section{Les bactéries considérées comme armes biologiques}

Ce bref historique nous rappelle que Bacillus anthracis n'est pas le seul micro-organisme susceptible d'être utilisé comme arme biologique. Théoriquement, plus de 
250 agents pathogènes pourraient servir les desseins des terroristes [3]. Parmi ceux-ci, on retrouve surtout des bactéries et des virus. Cependant, les bactéries ont la préférence des terroristes parce qu'elles sont en général plus faciles à obtenir et plus simples à propager.

Pour faire face à la menace des armes biologiques, les agences gouvernementales de plusieurs pays ont établi des listes hiérarchiques des armes potentielles. L'ordre de priorité des microorganismes varie un peu selon les perspectives des différents intervenants. À partir de critères déterminant le niveau de préparation et d'effort requis pour faire face à la menace microbiologique, les Centers for disease control (CDC) américains divisent les agents pathogènes en trois catégories [4] (Tableau 1). Les agents de catégorie A sont ceux qui pourraient entraîner des effets majeurs sur la santé publique et atteindre le plus de victimes. Les agents de catégorie $B$ ont un potentiel de dissémination à grande échelle, mais la morbidité et la mortalité qu'ils pourraient causer sont moins importantes que celles engendrées par les agents de catégorie A. Enfin, les microorganismes de la catégorie $C$ sont considérés comme constituant une menace émergente.

Il faut noter que plusieurs bactéries de catégorie B, tels les Vibrio cholerae, l'Escherichia coli entéropathogène, les shigelles et les salmonelles, sont surtout transmissibles par la nourriture et l'eau. Un contrôle plus strict de ces sources de contamination devra être entrepris afin d'éviter des désastres [5]. Bien que les maladies causées par ces pathogènes entériques soient en général limitées, elles peuvent être mortelles. II faut rappeler que l'attaque bioterroriste ayant fait le plus de victimes à ce jour, soit au moins 751 personnes, a été réalisée en 1984 par un groupe extrémiste religieux en Oregon par la contamination de bars à salades avec des salmonelles [6].

\section{Les tests actuels d'identification microbienne ne sont pas assez rapides}

Des modèles théoriques et l'expérience des lettres contaminées ont démontré que la rapidité d'intervention après une attaque biologique contribue à réduire significativement la morbidité et les coûts reliés à ces actes terroristes [7]. Malheureusement, la panique et le chaos engendrés par les alertes de l'automne 2001 ont prouvé que notre système de santé publique n'était pas prêt à faire face à des attaques utilisant des armes biologiques. Ce constat ne fait que révéler les limites du diagnostic microbiologique actuel. En effet, l'identification d'une bactérie prend encore aujourd'hui 36 à 72 heures. Pourtant, l'identification des microorga- nismes en moins d'une heure pourrait s'avérer une arme efficace contre le bioterrorisme [8, 9]. Enfin, il faut trouver une alternative à la culture des pathogènes dangereux, qui présente des risques pour le personnel des laboratoires cliniques [10] et requiert des installations spécialisées.

Les nouvelles technologies moléculaires, telle la PCR (polymerase chain reaction) en temps réel, permettent maintenant de diagnostiquer les infections en moins d'une heure, et ce directement à partir de l'échantillon clinique. À titre d'exemple, notre équipe a récemment publié des applications de la technologie de PCR en temps réel pour l'identification, en moins de 45 minutes, des femmes porteuses du streptocoque du groupe B au moment de l'accouchement [11, 12], et pour la détection rapide des $\varepsilon$. coli producteurs de toxines shiga directement à partir des selles [13]. Ces tests sont rapides, sensibles, spécifiques et ubiquitaires. La rapidité est assurée en combinant une méthode rapide ( $\leq 10$ minutes) de préparation de l'échantillon avec un appareil de PCR en temps réel permettant une amplification rapide de l'ADN ainsi que la mesure à chaque cycle d'un signal fluorescent spécifique à l'amplicon recherché.

La sensibilité de nos essais se situe en général à moins de 10 copies d'ADN cible par réaction PCR. La spécificité est évaluée en testant une batterie de souches couvrant les principales branches de l'arbre phylogénétique des bactéries, avec une représentation plus élevée des espèces proches de l'espèce ciblée. L'ubiquité de l'essai PCR est évaluée en testant plusieurs souches de l'espèce cible, provenant de différentes régions du globe et représentatives de la diversité génétique de cette espèce. Enfin, l'amplification et la détection du produit PCR étant réalisées dans un même tube qui n'est ouvert qu'une seule fois au début de l'essai, les chances de contamination par des amplicons précédemment obtenus sont minimes. Alors que les essais PCR classiques nécessitent jusqu'à une cinquantaine d'étapes techniques, ces nouvelles technologies permettent de réduire le nombre de manipulations à moins d'une dizaine.

La préparation d'échantillons soulève des problèmes particuliers en raison de la diversité des types d'échantillons cliniques dans lesquels il est possible de détecter les bactéries. Pour les bactéries de la catégorie A seulement, il existe plus d'une demi-douzaine de fluides biologiques qu'il faut pouvoir échantillonner, sans compter les nombreuses sources environnementales qu'il serait utile de pouvoir évaluer. Des systèmes microfluidiques automatisés, polyvalents et intégrés à la PCR sont actuellement en développement pour accomplir en quelques minutes cette tâche délicate [14]. 


\begin{tabular}{|c|c|c|c|c|c|c|c|c|c|c|}
\hline Bactérie & Maladie & Morbidité & Mortalité & P-D & P-P & $\begin{array}{l}\text { Perception } \\
\text { publique }^{1}\end{array}$ & $\begin{array}{l}\text { Préparation } \\
\text { spéciale }^{2}\end{array}$ & $\begin{array}{l}\text { Catégorie } \\
C^{3} C^{3}\end{array}$ & $\begin{array}{l}\text { Génome } \\
\text { séquencé }\end{array}$ & $\begin{array}{l}\text { Structure } \\
\text { du génome }\end{array}$ \\
\hline $\begin{array}{l}\text { Bacillus } \\
\text { anthracis }\end{array}$ & Charbon & ++ & +++ & +++ & - & +++ & +++ & A & 0 & $\begin{array}{l}1 \text { chromosome } \\
2 \text { plasmides }\end{array}$ \\
\hline Yersinia pestis & $\begin{array}{l}\text { Peste } \\
\text { pulmonaire }\end{array}$ & ++ & +++ & ++ & ++ & ++ & +++ & A & 0 & $\begin{array}{l}1 \text { chromosome } \\
3 \text { plasmides }\end{array}$ \\
\hline $\begin{array}{l}\text { Clostridium } \\
\text { botulinum }\end{array}$ & Botulisme & ++ & +++ & ++ & - & ++ & +++ & A & $\varepsilon$ & 1 chromosome \\
\hline $\begin{array}{l}\text { Francisella } \\
\text { tularensis }\end{array}$ & Tularémie & ++ & ++ & ++ & - & + & +++ & A & $\varepsilon$ & 1 chromosome \\
\hline $\begin{array}{l}\text { Coxiella } \\
\text { burnetii }\end{array}$ & Fièvre $\rho$ & + & + & ++ & - & + & ++ & B & $\varepsilon$ & l chromosome \\
\hline Brucella spp. & Brucellose & + & + & ++ & - & + & ++ & B & 0 & 2 chromosomes \\
\hline $\begin{array}{l}\text { Burkholderia } \\
\text { mallei }\end{array}$ & Morve & ++ & +++ & ++ & $+/-$ & - & ++ & B & $\varepsilon$ & 2 chromosomes \\
\hline $\begin{array}{l}\text { Burkholderia } \\
\text { pseudomallei }\end{array}$ & Mélioïdose & + & + & ++ & - & - & ++ & B & 0 & 2 chromosomes \\
\hline $\begin{array}{l}\text { Chlamydophila } \\
\text { psittaci }\end{array}$ & Psittacose & + & + & ++ & - & - & ++ & B & N & 1 chromosome \\
\hline $\begin{array}{l}\text { Rickettsia } \\
\text { prowazekii }\end{array}$ & Typhus & + & + & ++ & - & - & + & B & 0 & 1 chromosome \\
\hline Vibrio cholerae & Choléra & + & + & ++ & $+/-$ & +++ & + & B & 0 & 2 chromosomes \\
\hline $\begin{array}{l}\text { Escherichia coli } \\
\text { 0157:H7 }\end{array}$ & $\begin{array}{l}\text { Maladie du } \\
\text { hamburger }\end{array}$ & + & + & ++ & + & + & + & B & 0 & 1 chromosome \\
\hline Shigella spp. & Shigellose & + & + & ++ & + & + & + & B & $\varepsilon$ & $\begin{array}{l}1 \text { chromosome } \\
\text { I plasmide }\end{array}$ \\
\hline Salmonella spp. & $\begin{array}{l}\text { Salmonellose } \\
\text { typhoïde }\end{array}$ & + & + & ++ & + & + & + & B & 0 & $\begin{array}{l}1 \text { chromosome } \\
0-2 \text { plasmides }\end{array}$ \\
\hline $\begin{array}{l}\text { Mycobacterium } \\
\text { tuberculosis } \\
\text { multirésistant }\end{array}$ & $\begin{array}{l}\text { Tuberculose } \\
\text { multirésistant }\end{array}$ & & + & ++ & + & ++ & ++ & C & 0 & 1 chromosome \\
\hline
\end{tabular}

Tableau I. Propriétés des armes bactériologiques, listées de la menace la plus importante (+++) à la menace la plus faible (-). P-D: potentiel pour production et dissémination dans des quantités suffisantes pour affecter une grande population, déterminé à partir de la disponibilité, des besoins pour la culture, de la voie d'infection la plus probable et de la stabilité environnementale; P-P: transmissibilité de personne à personne; 0 : oui; $N$ : non; $\varepsilon$ : en cours. 1. Perception publique du danger de ces infections; 2. Niveau de préparation spéciale requis par les laboratoires de santé publique pour faire face à ces infections; 3. Catégorie de priorité selon les CDC (centers for disease control). A: agents ayant le plus de potentiel néfaste; $B$ : agents à potentiel important mais perçus comme plus simples à contrôler; $C$ : agents émergents; 4 . Structure minimale requise pour servir d'arme biologique pleinement virulente (adapté de [4]). 


\section{Le choix d'une cible pour le diagnostic moléculaire}

L'identification moléculaire d'un pathogène repose sur la détection d'un gène cible (Figure 1) qui doit pouvoir être relié spécifiquement à tous les représentants de l'espèce microbienne recherchée. Traditionnellement, le gène codant pour l'ARN ribosomal 165 a été le plus utilisé pour cette tâche. D'autres gènes conservés dans l'évolution, tels tuf, recA, atpD, grok et dnaK, sont également utilisables à ces fins [9]. $\mathrm{Si}$, en plus, ce gène conservé est essentiel à la survie de la bactérie, on minimise la possibilité d'avoir des faux négatifs avec des organismes modifiés génétiquement. En revanche, ces gènes conservés ne permettent pas toujours de distinguer les souches virulentes des souches avirulentes. C'est le cas par exemple pour $B$. anthracis qui doit posséder deux plasmides, $\mathrm{p} 0 \mathrm{X} 1$ et $\mathrm{p} 0 \mathrm{X} 2$, pour causer la maladie du charbon. L'analyse des gènes de virulence de ces plasmides a permis de déterminer que l'échec des attaques biologiques de la secte Aum au Japon était dû à l'utilisation d'une souche vaccinale non virulente [15]. De plus, en ciblant des gènes requis pour la pathogenèse, on peut éventuellement identifier le transfert de ces facteurs de virulence à d'autres espèces. La détection de gènes conférant la résistance aux antibiotiques assurerait un choix thérapeutique optimal d'autant plus important que les chercheurs soviétiques auraient créé une souche de Yersinia pestis capable de résister à plus de 16 antibiotiques différents [16]. La diversité des gènes de résistance pose des limites qu'il faudra résoudre par I'utilisation de futures technologies permettant l'analyse simultanée de multiples cibles génétiques. Les possibilités de manipulations génétiques, même de gènes essentiels comme celui de l'ARN ribosomal 16S [17], nous indiquent que l'analyse de multiples cibles et même de génomes entiers est la voie de l'avenir.

\section{Un essai rapide pour Bacillus anthracis}

Nous avons démontré l'efficacité de notre approche pour le choix des cibles en développant un essai moléculaire rapide pour l'identification de Bacillus anthracis [18]. L'analyse de séquences des plasmides $p 0 X 1$ et $\mathrm{p} 0 \times 2$ de plusieurs souches de $B$. anthracis a permis de choisir des séquences conservées dans des gènes essentiels à la virulence. L'analyse des séquences de gènes chromosomiques essentiels a permis d'identifier un polymorphisme unique à $B$. anthracis. Nous avons développé un essai PCR rapide $(<1$ heure) qui identifie simultanément par fluorescence les deux cibles plasmidiques ainsi qu'un gène chromosomique de B. anthracis. Cet essai a permis la détection sensible, spécifique et ubiquitaire des cellules végétatives ainsi que des spores de $B$. anthracis ajouté à différents types d'échantillons biologiques. La sensibilité analytique de l'essai réalisé à partir d'écouvillons nasaux inoculés avec $B$. anthracis est de moins de 10 unités formant des colonies par réaction PCR. La technologie utilisée dans le développement de cet essai devrait être adaptée à d'autres agents biologiques.

\section{Perspectives}

Pour paraphraser La Fontaine, nous n'en mourrons pas tous, mais nous sommes tous atteints, à des degrés divers, par les événements de l'automne 2001. Le besoin urgent de répondre à la menace des armes bactériologiques nous force à améliorer les moyens diagnostiques expérimentaux existants pour les faire entrer en appli-

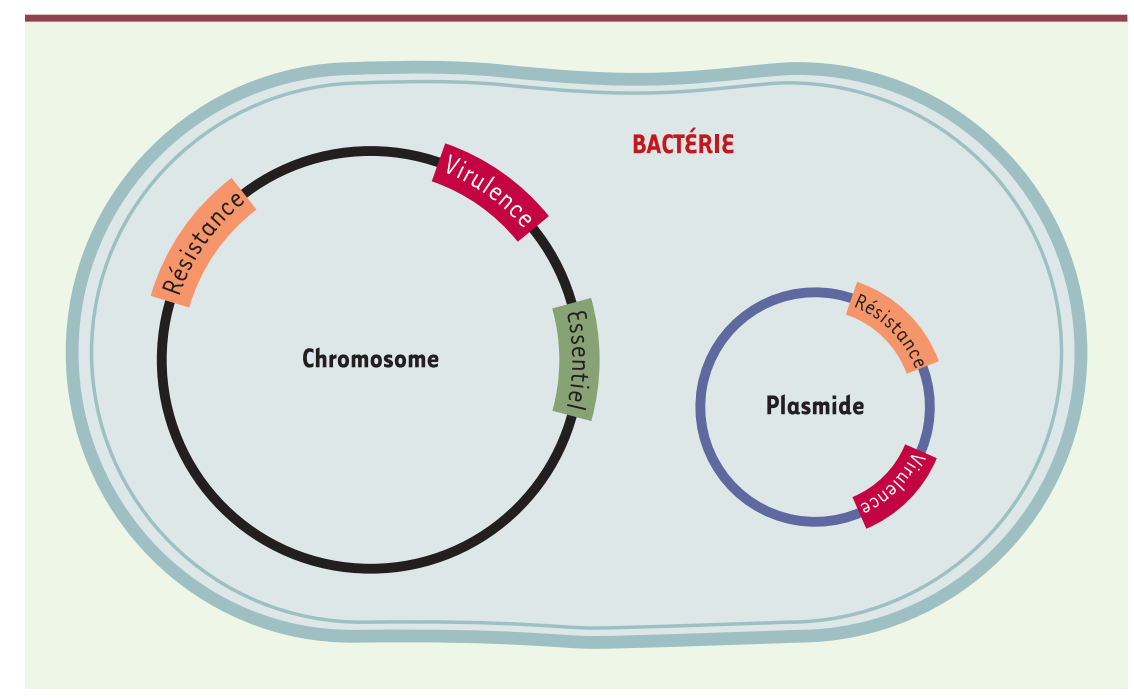

Figure 1. Types de cibles génétiques permettant l'identification des armes bactériologiques. En ciblant un gène essentiel (en vert) pour la survie de la bactérie, les chances de détecter l'ensemble des représentants d'une même espèce sont optimisées, tandis que les risques d'avoir des faux négatifs avec des souches manipulées génétiquement sont réduits. La détection simultanée de gènes requis pour la virulence (en rouge) assure l'identification des souches pathogènes et pourrait donner des indications sur le transfert de ces gènes de virulence à d'autres espèces. L'identification de gènes de résistance (en orange) sert à guider l'intervention thérapeutique. Enfin, la détection simultanée de cibles situées sur différents éléments génétiques - chromosome(s) (trait noir) ou plasmide(s) (trait bleu) - procure des informations sur des réarrangements génétiques potentiels de l'agent pathogène. 
cation clinique le plus rapidement possible. En revanche, il nous apparaît tout aussi important de pouvoir diagnostiquer toutes les infections en moins d'une heure, de manière à fournir au clinicien les résultats de la microbiologie en même temps que les analyses biochimiques, sanguines et radiologiques. Le médecin pourra alors poser un diagnostic non pas présomptif, comme il est trop souvent obligé de le faire maintenant, mais un diagnostic enfin basé sur des preuves obtenues au laboratoire. Cela devrait révolutionner la pratique médicale, car le traitement des maladies infectieuses sera basé sur l'identification précise du pathogène et des gènes de résistance au moment où le clinicien voit son patient. Ces tests d'identification en temps réel devraient non seulement favoriser une thérapie optimale, mais aussi contribuer à réduire l'impact de la résistance microbienne [8]. Cette fusion du diagnostic et de la thérapie, nous l'appelons théranostic moléculaire [9]. $\diamond$

\section{REMERCIEMENTS}

Nous tenons à remercier Natalie Clairoux, Éric Leblanc et François J. Picard pour leurs judicieux commentaires. L'équipe de diagnostic moléculaire du CRI est supportée par la subvention PA-15586 des Instituts Canadiens de Recherche en Santé et Infectio Diagnostic (IDI) Inc.

\section{SUMMARY}

\section{Genomics and bioterrorism}

The use of biological weapons has been recorded throughout history. However, the anthrax-tainted letters of the fall of 2001 caused shock and panic in several countries. Knowledge of the principal bacteriological weapons allows design of novel rapid DNA-based diagnostic tests that should help defuse the impact of future bioterrorist attacks. Less than one-hour real-time PCR identification of bacteria and their associated antibiotic resistance genes will revolutionize the practice of medicine. $\nabla$

\section{RÉFÉRENCES}

1. Redmond C, Pearce MJ, Manchee RJ, Berdal BP. Deadly relic of the Great War. Nature 1998; 393: 747-8.

2. Christopher GW, Cieslak TJ, Pavlin JA, Eitzen EM, Jr. Biological warfare. A historical perspective. JAMA 1997; 278: 412-7.

3. Montplaisir S. Bioterrorisme : L'heure est-elle seulement pour Bacillus anthracis? Les Sélections de médecine/sciences 2001 ; $19: 10-3$.

4. Rotz LD, Khan AS, Lillibridge SR, Ostroff SM, Hughes JM. Public health assessment of potential biological terrorism agents. Emerg Infect Dis $2002 ; 8$ : 225-30.

5. Sobel J, Khan AS, Swerdlow DL. Threat of a biological terrorist attack on the US food supply : the CDC perspective. Lancet 2002 ; $359: 874-80$.

6. Torok TJ, Tauxe RV, Wise RP, et al. A large community outbreak of salmonellosis caused by intentional contamination of restaurant salad bars. JAMA 1997 ; $278:$ 389-95.

7. Kaufmann AF, Meltzer MI, Schmid GP. The economic impact of a bioterrorist attack : are prevention and postattack intervention programs justifiable? Emerg Infect Dis 1997 ; 3 : 83-94.

8. Boissinot M, Bergeron MG. Toward rapid real-time molecular diagnostic to guide smart use of antimicrobials. Curr Opin Microbiol 2002 ; 5 : 478-82.

9. Picard FJ, Bergeron MG. Rapid molecular theranostics in infectious diseases. Drug Discov Today $2002 ; 7$ : 1092-101.

10. Shapiro DS, Schwartz DR. Exposure of laboratory workers to Francisella tularensis despite a bioterrorism procedure. J Clin Microbiol 2002 ; 40 : 2278-81.
11. Bergeron MG, Ke D, Menard $C$, et al. Rapid detection of group B streptococci in pregnant women at delivery. $N$ Engl J Med 2000; 343: 175-9.

12. Ke D, Menard C, Picard FJ, et al. Development of conventional and real-time PCR assays for the rapid detection of group $B$ streptococci. Clin Chem 2000; 46: 324-31.

13. Belanger SD, Boissinot $M$, Menard C, Picard FJ, Bergeron MG. Rapid detection of Shiga toxinproducing bacteria in feces by multiplex PCR with molecular beacons on the smart cycler.

J Clin Microbiol 2002; 40: 1436-40.

14. Taylor MT, Belgrader $P$, Furman BJ, et al. Lysing bacterial spores by sonication through a flexible interface in a microfluidic system. Anal Chem 2001; 73: 492-6.

15. Keim P, Smith KL, Keys C, et al. Molecular investigation of the Aum Shinrikyo anthrax release in Kameido, Japan. J Clin Microbiol 2001; 39: 4566-7.

16. Niiler $\varepsilon$. Bioterrorismbiotechnology to the rescue? Nat Biotechnol 2002; 20: 21-5.

17. Asai T, Zaporojets D, Squires C, Squires CL. An Escherichia coli strain with all chromosomal rRNA operons inactivated: complete exchange of rRNA genes between bacteria. Proc Natl Acad Sci USA 1999; 96: 1971-6.

18. Leblanc $\varepsilon$, Vaillancourt $M$, Boissinot $M$, et al. Less than one hour detection of Bacillus anthracis spores and vegetative cells from clinical specimens by fluorescence-based PCR. ASM, $102^{\text {nd }}$ general meeting. Salt Lake City, Utah : ASM, 2002.

\section{TIRÉS À PART}

M. Boissinot
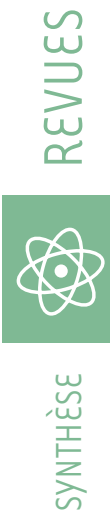